\title{
ON CONFORMAL MAPPING OF A MULTIPLY-CONNECTED DOMAIN ONTO A CANONICAL COVERING SURFACE, II
}

\author{
By Hisao Mizumoto
}

\section{§1. Introduction.}

In our previous paper [1] we concerned ourselves with a conformal mapping of a multiply-connected planar domain of finite connectivity each boundary component of which is a continuum onto a covering surface of annular type cut along concentric circular slits (cf. $\$ 2$ of [1]). And we obtained a procedure of constructing the mapping function by an extremal method.

In the present note we will concern ourselves with a conformal mapping onto another canonical covering surface. We shall obtain the following result: There exists an analytic function which maps a multiply-connected domain of finite connectivity each boundary component of which is a continuum onto a covering surface of circular type cut along concentric circular slits (cf. \$2). If we preassign the rotation number about the origin of the image of every boundary component of the original domain and the zero points with their orders, the mapping function is determined uniquely except an entire linear transformation on the basic plane of the image (THEOREM in $\S 3$ ). It will be shown that this result is obtained by using the one in [1].

\section{§2. Preliminaries.}

Let $F$ be a finitely-sheeted covering surface lying over the $w$-plane whose boundary $\Gamma$ consists of $N$ continua

$$
\Gamma_{3} \quad(j=1, \cdots, N) .
$$

If $F$ is bounded and such that there exists a point of $F$ but no point of $\Gamma$ on a point $a$, then we call $F$ a finitely-sheeted bounded covering surface with a as an inner point.

Let $G$ be a finitely-sheeted bounded covering surface with $a$ as an inner point each boundary component of which

$$
\text { A, } \quad(j=1, \cdots, N)
$$

has, as the projection onto the basic plane, a circle or a circular slit centred at $a$. Then we call $G$ a covering surface of circular type cut along concentric circular slits centred at $a^{1)}$.

Received September 14, 1959.

1) It is permitted that there is no concentric circular slit. 
Let $F$ be a finitely-sheeted bounded covering surface with $a$ as an inner point. Let $F_{r}$ be a subset of $F$ which is obtained by deleting all portions of $F$ lying over

$$
|w| \leqq r \quad(r>0),
$$

where $r$ should be chosen so that there is no point of $\Gamma$ lying over $|w| \leqq r$. Further let

$$
\Gamma_{\jmath}^{*} \quad(j=1, \cdots, N)
$$

be simple analytic closed curves on $F_{r}$ homotopic to $\Gamma_{\jmath}$ on $F_{r}$, respectively.

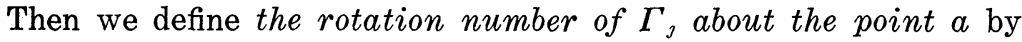

$$
\frac{1}{2 \pi} \int_{\Gamma j^{*}} \arg (z-a)=\nu_{\jmath} \quad(j=1, \cdots, N) .
$$

The value on the left-hand side of (1) does not depend on a particular choice of $F_{r}$ and $\Gamma_{j}{ }^{*}$.

The rotation number about the point $a$ of the union of some boundary components is defined by the sum of their rotation numbers of each boundary component about $a$.

\section{§3. Theorem.}

The purpose of the present note is to verify the following fundamental theorem which shows that there exists an analytic function mapping a multiplyconnected domain of finite connectivity onto a covering surface of circular type cut along concentric circular slits.

THEOREM. Let $B$ be a multiply-connected domain of finite connectivity on the z-plane. We suppose that each component $C_{\jmath}(j=1, \cdots, N)$ of its boundary $C$ is a continuum. Then $B$ can be conformally mapped onto a covering surface of circular type $G$ cut along concentric circular slits centred at the origin. Further we can preassign the rotation number about the origin of the image of each boundary component and the zero points with their orders arbitrarily under the condition that the sum of the rotation numbers minus the total order of the zeros is equal to zero (except the case where there exists no zero). If we preassign the rotation number about the origin of the image of each boundary component and the zero points with their orders, the mapping function

$$
w=\Psi(z)
$$

is uniquely determined under an additional condition $\Psi\left(z_{0}\right)=1$ where $z_{0}$ is an arbitrarily preassigned point on $B$ but is not a zero point.

Proof. Let the rotation number about the origin of the image $C_{\jmath}(j=1$, 
$\cdots, N$ ) be equal to

$$
\nu_{j} \quad(j=1, \cdots, N)
$$

and let $z_{k}\left(k=1, \cdots, N^{\prime}\right)$ be the zero point with its order

$$
\mu_{k} \quad\left(k=1, \cdots, N^{\prime}\right) .
$$

By the assumption of the theorem they must satisfy the condition

$$
\sum_{j=1}^{N} \nu_{\jmath}-\sum_{k=1}^{N^{\prime}} \mu_{k}=0
$$

Let $\left\{\varepsilon_{m}\right\}_{m=1}^{\infty}$ be a monotone decreasing sequence of positive numbers which converges to zero. Let $\varepsilon_{1}$ be chosen sufficiently small so that the closed disks

$$
\left|z-z_{k}\right| \leqq \varepsilon_{1} \quad\left(k=1, \cdots, N^{\prime}\right)
$$

are contained in $B$ and are mutually disjoint. Let $B^{m}$ be the subdomain of $B$ which is obtained by deleting the closed disks

$$
\left|z-z_{k}\right| \leqq \varepsilon_{m} \quad\left(k=1, \cdots, N^{\prime}\right)
$$

and let $C_{k}^{m}\left(k=1, \cdots, N^{\prime}\right)$ be the boundary components $\left|z-z_{k}\right|=\varepsilon_{m}$ of $B^{m}$. Then each $B^{m}(m=1,2, \cdots)$ is a subdomain of $B$ whose boundary consists of a finite number of boundary components $C_{\jmath}(j=1, \cdots, N)$ and $C_{k}^{m}\left(k=1, \cdots, N^{\prime}\right)$. Let $B^{m *}$ be a subdomain of $B^{m}$ whose boundary $C^{m *}$ consists of components $C_{j}{ }^{*}(j=1, \cdots, N)$ and $C_{k}^{m}\left(k=1, \cdots, N^{\prime}\right)$, where $C_{\jmath}{ }^{*}$ is a simple analytic closed curve homotopic to $C_{\jmath}$ in $B^{1}$.

By Theorem 2 of $[1]$, there exists an analytic function $w=\Phi_{m}(z)$ which maps $B^{m}$ onto a covering surface of annular type $G^{m}$ cut along concentric circular slits centred at the origin (cf. $\$ 2$ of [1]) and satisfies

$$
\begin{aligned}
& \frac{1}{2 \pi} \int_{C_{j^{*}}} d \arg \Phi_{m}(z)=\nu_{\jmath} \quad(j=1, \cdots, N), \\
& \frac{1}{2 \pi} \int_{C_{k}^{m}} d \arg \Phi_{m}(z)=-\mu_{k} \quad\left(k=1, \cdots, N^{\prime}\right) \\
& \Phi_{m}\left(z_{0}\right)=1 \text {. }
\end{aligned}
$$

We denote the image curves of $C_{\jmath}(j=1, \cdots, N)$ and $C_{k}^{m}\left(k=1, \cdots, N^{\prime}\right)$ by $\Lambda_{\jmath}^{m}$ and $\Lambda_{k}^{m}$, respectively. The sequence $\left\{\Phi_{m}\right\}_{m=1}^{\infty}$ is obviously normal, i.e., it contains a subsequence which converges uniformly on every compact subset of $B-\left\{z_{k}\right\}_{k=1}^{N \prime}$. Without loss of generality, we may suppose that $\left\{\Phi_{m}\right\}_{m=1}^{\infty}$ does so and let $\Psi$ be the limiting function.

Next we shall show that the function $\Psi$ thus obtained is the desired mapping function. Obviously $\Psi$ is regular on $B-\left\{z_{k}\right\}_{k=1}^{N^{\prime}}$ and $\Psi\left(z_{0}\right)=1$. Further, since $\Phi_{m}$ converges to $\Psi$ uniformly on $C_{\jmath}{ }^{*}(j=1, \cdots, N)$ and $C_{k}^{1}\left(k=1, \cdots, N^{\prime}\right)$, we have 


$$
\begin{array}{ll}
\int_{C_{j^{*}}} d \arg \Psi=\lim _{m \rightarrow \infty} \int_{C_{j^{*}}} d \arg \Phi_{m}=2 \pi \nu_{j} & (j=1, \cdots, N), \\
\int_{C_{k}^{1}} d \arg \Psi=\lim _{m \rightarrow \infty} \int_{C_{k}^{1}} d \arg \Phi_{m}=-2 \pi \mu_{k} & \left(k=1, \cdots, N^{\prime}\right) .
\end{array}
$$

Since $\Psi$ is single-valued, regular and bounded in $0<\left|z-z_{k}\right|<\varepsilon_{1}$, we see by Riemann's theorem for removable singularity that $z_{k}$ is a regular point of $\Psi$ $\left(k=1, \cdots, N^{\prime}\right)$. Obviously, $z_{k}$ is the zero point of $\Psi$ and, by (2), its order is $\mu_{k}\left(k=1, \cdots, N^{\prime}\right)$.

We have to show that the projection onto the $w$-plane of the image $A_{j}$ of each boundary component $C_{3}$ by $\Psi$ lies on a circle centred at the origin $(j=1$, $\cdots, N)$. The domain $B^{1}$ can be mapped one-to-one conformally onto $G^{1}$ by $\Psi_{1}$. Therefore the function $\Phi_{m} \circ \Phi_{1}^{-1}$ defines the conformal mapping of $G^{1}$ into $G^{m}$ which maps $\Lambda_{j}^{1}$ onto $\Lambda_{j}^{m}(j=1, \cdots, N ; m=1,2, \cdots)$. Since $\Lambda_{j}^{1}$ and $\Lambda_{j}^{m}$ lie on circles centred at the origin, respectively, $\Phi_{m} \circ \Phi_{1}^{-1}$ are analytically continuable through $\Lambda_{j}^{1}(j=1, \cdots, N)$ by means of the Schwarz's reflexion principle. Then we see that the sequence $\left\{\Phi_{m} \circ \Phi_{1}^{-1}\right\}_{m=1}^{\infty}$ converges uniformly on the closure $\bar{G}^{1}$ and therefore the image $\Lambda_{j}$ of $\Lambda_{j}^{1}$ by its limit function $\Psi_{\circ} \Phi_{1}^{-1}$ lies on a circle centred at the origin as the limit of $\left\{\Lambda_{j}^{m}\right\}_{m=1}^{\infty}$.

It remains only to see that the mapping function $\Psi$ is unique. Let $\Psi^{*}$ be another one and $f=\lg \Psi^{*}-\lg \Psi$. We can easily see that $f$ is single-valued and regular on $B$ and maps each boundary component $C_{\jmath}$ onto a bounded vertical slit. Therefore $f$ is bounded. But this is impossible unless $f \equiv$ const. Since $f\left(z_{0}\right)=0$, we conclude that $f \equiv 0$.

\section{REFERENCE}

[1] Mizumoto, H., On conformal mapping of a multiply-connected domain onto a canonical covering surface. Kōdai Math. Sem. Rep. 10 (1958), 177-188.

Department of Mathematics, Tokyo Institute of Technology. 